\title{
A Single Tertiary Center Outcomes on Cannulation Strategies and Extracorporeal Membrane Oxygenation in the Treatment of Respiratory Failure During COVID- 19 Infection
}

\author{
(- Mustafa Ozer Ulukan, • A Atalay Karakaya, • Yahya Yildiz*, ๑ Didem Melis Oztas**,

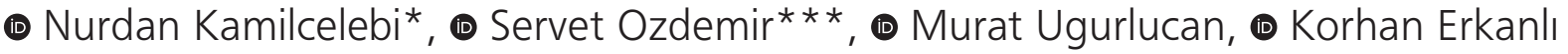 \\ Istanbul Medipol University Faculty of Medicine, Department of Cardiovascular Surgery, Istanbul, Turkey \\ *Istanbul Medipol University Faculty of Medicine, Department of Anesthesiology and Reanimation, Istanbul, Turkey \\ **University of Health Sciences Turkey, Bagcllar Training and Research Hospital, Clinic of Cardiovascular Surgery, Istanbul, Turkey \\ ***Bakırköy Training and Research Hospital, Clinic of Thoracic Surgery, Istanbul, Turkey
}

\section{Abstract}

\begin{abstract}
Aim: Extracorporeal membrane oxygenation (ECMO) is an important option for the management of severe acute respiratory distress syndrome (ARDS) in Coronavirus disease-2019 (COVID-19) cases. We aimed to present our experiences of ECMO in patients with respiratory failure secondary to COVID-19.

Methods: Data of 22 consecutive COVID-19 patients with severe respiratory failure whom were supported with ECMO were collected from computer-based hospital software retrospectively. Patients were treated in a single medical center between April 23, 2020 and February 14, 2021. Patients were analyzed from the points of laboratory and inflammatory markers, ventilation and ECMO features.

Results: The ages of patients were between 30 and 69 years (mean age: 56.3 \pm 10.63 ). All patients were under maximum ventilator support, with the prone position. All patients had elevated levels of inflammatory indicators as D-dimer and ferritin. The mean level of ferritin was 1,564 $\pm 1,611 \mathrm{ng} / \mathrm{mL}$. D-dimer value was maximum $10.000 \mathrm{mg} / \mathrm{mL}$ (mean: 5,215 $\pm 3,104$ ), CRP increased to $177 \mathrm{mg} / \mathrm{L}$ (mean: 159 \pm 71 ). Percent of lymphocytes decreased as low as $2 \%$ (mean: 4.16 \pm 2.10 ). The mean duration of veno-arterial (VA) ECMO was $1.6 \pm 0.94$ days whereas, for veno-venous (VV) ECMO, it was $10.05 \pm 5$ days. VA ECMO was decided due to cardiovascular collapse. Four patients with VA ECMO survived a maximum of 3 days. Four of (22.22\%) of 18 VV ECMO supported patient's blood gas values were at normal ranges, 3 of them needed tracheostomy, and all of could be discharged from the hospital.
\end{abstract}

Conclusion: Although, ECMO support for severe respiratory failure patients with COVID-19 is more challenging than regular ECMO applications, especially VV ECMO usage should be reminded as a remedy.

Keywords: COVID-19, extracorporeal membrane oxygenation, multi-organ failure

\section{Introduction}

The novel Coronavirus disease-2019 (COVID-19) induced disease (COVID-19) causes a rapidly evolving pandemic infection (1). The clinical findings of COVID-19 include high fever, dry cough, fatigue, pain especially at the back, diarrhea, and bilateral pneumonia, which can expand into acute respiratory distress syndrome (ARDS), metabolic acidosis, septic shock, coagulopathy, and hemorrhagic-septic multi-organ failure. Cytokine storm and hyper inflammation appear to be major components of severe COVID-19 pneumonia and multi-organ failure $(2,3)$. The duration and severity of COVID-19 pneumonia and the time for improvement are currently not defined (4). There is not any approved treatment protocol except supportive care (5). The COVID-19 has begun to spread in Turkey by March 2019 (6).

Address for Correspondence: Mustafa Ozer Ulukan, Istanbul Medipol University Faculty of Medicine, Department of Cardiovascular Surgery, Istanbul, Turkey Phone: +90 2124607000 E-mail: ozerulukan@hotmail.com ORCID: orcid.org/0000-0001-9919-8392 Received: 02.03.2021 Accepted: 12.04.2021

'Copyright 2021 by The Medical Bulletin of istanbul Haseki Training and Research Hospital The Medical Bulletin of Haseki published by Galenos Yayınevi. 
The percentage of hospitalized patients who required extracorporeal membranous oxygenation (ECMO) due to COVID-19 associated ARDS was reported as 2.8\% on preliminary reports from China (7). The treatment of severe respiratory failure with ECMO needs expertise and skills (8). The number of patients who present to hospitals for ECMO annually is positively associated with the survival rate of the patients. WHO interim guidelines on the management of suspected COVID-19 recommend supporting with veno-venous (VV) ECMO to appropriate patients with ARDS related to COVID-19 in skilled centers with sufficient case volumes and clinical expertise (4). COVID-19 pneumonia in severe cases with low blood oxygen saturation may disturb hemodynamic values and causes multi-organ failure, so in these cases; ECMO may be the last life-saving tool. But of course, it is not the main treatment method of conventional ARDS as moderate positive end-expiratory pressure (PEEP), low tidal volumes and restricting plateau airway, and mild hypercapnia are among the first order management strategies. The addition of ECMO to treatment was based on the severity of respiratory failure despite protective ventilation and prone position of patients in deterioration of clinical findings despite appropriate treatment $(7,9)$.

In the current research, we present our experiences with VV and veno-arterial (VA) ECMO support in the managing of patients with COVID-19.

\section{Methods}

\section{Study Design}

Ethics committee approval was obtained from the Istanbul Medipol University Ethics Committee (date: 02.18.2021, approval number: 179) for the retrospective analysis of the respiratory failure of COVID-19 patients treated with ECMO. All the patients were informed at the time of hospitalization and consent forms were signed by the patient(s) as well as a legally authorized representative.

The selected patients for ECMO application were evaluated by a trained team consisting of pulmonary diseases, Anesthesiology, Intensive Care, Cardiac Surgery, and Infectious Diseases experts.

Data were collected retrospectively through computer based hospital system from 22 patients with COVID-19 who had severe respiratory failure and were supported with ECMO. The diagnosis of COVID-19 confirmed using polymerase chain reaction (PCR). Patients were treated in a single medical center from April 23, 2020 to February 04, 2021. Regarding VV or VA ECMO, patients were divided into two groups as Group 1 and Group 2. Patients' comorbidities were evaluated regarding Charlson Comorbidity index (CCl) (10). Aggressive mechanical ventilation with a peak airway pressure of higher than
$30 \mathrm{~cm} \mathrm{H}_{2} \mathrm{O}$ and fraction of inspired oxygen $\left(\mathrm{FiO}_{2}\right)$ higher than 0.8$)$ for more than one week was accepted as an indication for ECMO. Severe comorbidity as myocardial infarction, congestive heart failure, peripheral vascular disease, cerebrovascular disease, dementia, chronic pulmonary disease, connective tissue diseases, previous ulcer diseases, liver diseases, hemiplegia, severe renal diseases, diabetes with end-organ damage, any tumor state, leukemia, lymphoma, metastatic solid tumor, AIDS and multi-organ failure, sepsis, and age above 75 years were used as contraindications for VV or VA ECMO application.

\section{ECMO Application}

All patients had severe lung damage. They were fully anesthetized and received mechanical ventilation. So, there was no need for local anesthesia for peripheral cannulation. Cannulation strategies for VA ECMO were from the femoral artery and vein whereas dual-stage right atrium-to-inferior vena cava cannula with the aid of echocardiogram or femoral and internal jugular veins cannulations were for $\mathrm{VV}$ ECMO. The cannulation techniques and the decision of weaning off ECMO were shown in the research flow diaphragm.

\section{Statistical Analysis}

The data analysis was arranged by software Statistical Package for Social Sciences, SPSS 20.0. Descriptive statistics and percentages for categorical variables, means, and standard deviation were used to evaluate the clinical and demographic characteristics of the patients. Categorical values were evaluated with "the chi-square test," and parametric values were evaluated with "independent samples t-test". Correlation analysis was evaluated by Spearman rank and Pearson correlation coefficients. $\mathrm{P}<0.05$ was considered statistically significant.

\section{Results}

Care with ECMO was performed in 22 consecutive patients between the ages of 30 and 69 years (mean age, $56.3 \pm 10.63)$. While 16 of patients were male $(72.72 \%)$, 6 of the patients were female $(27.27 \%$ ) (Table 1$)$. In the medical history of patients, there was no known history of hypertension, diabetes mellitus, asthma, chronic obstructive pulmonary disease, chronic atherosclerotic heart disease, venous thrombosis, or chronic renal disease. $\mathrm{CCl}$ was lower than 3 in all of the patients.

All patients reached maximum ventilator support, with $100 \% \mathrm{FiO}_{2}$ and placed in a prone position. Pre-ECMO; all the patients required high doses of vasopressors, mean inotropic scores reaching $36.4 \pm 5.9$. BUN and creatinine levels were within the normal range at the time of hospitalization. All patients had meaningfully elevated 
Table 1. Demographic data and ECMO duration

\begin{tabular}{|c|c|c|c|c|}
\hline & & $\begin{array}{l}\text { Group 1 } \\
\text { (VV ECMO) } \\
(\mathrm{n}=18)\end{array}$ & $\begin{array}{l}\text { Group } 2 \\
\text { (VA ECMO) } \\
(\mathrm{n}=4)\end{array}$ & $\begin{array}{l}\text { Total } \\
(\mathrm{n}=22)\end{array}$ \\
\hline \multicolumn{2}{|l|}{ Age } & $56.7 \pm 10.7$ & $59 \pm 11.1$ & $56.3 \pm 10.6$ \\
\hline \multirow{2}{*}{ Sex } & Male & $13(72.2 \%)$ & $3(75 \%)$ & $16(72.7 \%)$ \\
\hline & Female & $5(27.7 \%)$ & $1(25 \%)$ & $6(27.2 \%)$ \\
\hline \multicolumn{2}{|c|}{ Weaned of ECMO } & $4(22.2 \%)$ & - & $4(18.1 \%)$ \\
\hline \multicolumn{2}{|c|}{$\begin{array}{l}\text { Mean duration of } \\
\text { ECMO/day }\end{array}$} & $10 \pm 5$ & $1.6 \pm 0.9$ & $8.5 \pm 5.5$ \\
\hline \multicolumn{2}{|c|}{$\begin{array}{l}\text { Erythrocyte infusion } \\
\text { per day }(\mathrm{mL})\end{array}$} & $437 \pm 29$ & $600 \pm 74$ & $466 \pm 76$ \\
\hline
\end{tabular}

levels of inflammatory indicators, such as D-dimer and ferritin, before ECMO use. The mean ferritin level was 1.564.08 $\pm 1.611 .90 \mathrm{ng} / \mathrm{mL}$ (minimum: 338, maximum: 7.000). D-dimer value was a maximum of $10.000 \mathrm{mg} /$ $\mathrm{mL}$ (mean; 5215.15 \pm 3104.75$)$, CRP increased to a

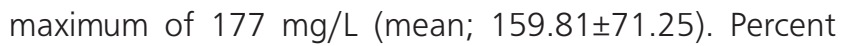
of lymphocytes decreased as low as 2\% (4.16 2.10$)$. The mean time from intubation to ECMO was found as $9.33 \pm 4.28$ days. The mean duration of VA ECMO was $1.6 \pm 0.94$ days whereas, for $\mathrm{VV} E C M O$, it was $10.05 \pm 5$ days. VA ECMO was decided due to cardiovascular collapse despite high doses of inotropic support (mean inotrope score: $64.2 \pm 16.5)$. Four of the patients with VA ECMO survived a maximum of 3 days and unfortunately lost. These patients needed high doses of inotropic agents and a mean of $600 \pm 74 \mathrm{~mL}$ of erythrocyte infusion per day. The needed rate of erythrocyte infusion for $\mathrm{VV}$ ECMO patients was $437 \pm 29 \mathrm{~mL}$ per day. Four of 18 patients with VV ECMO support could be weaned off ECMO after gradually decreasing flow and oxygen support from ECMO. Unfortunately, the remaining patients with VV ECMO were lost while still on ECMO. Among the patients who could be weaned off ECMO, one of them died due to cardiac rhythm disorders and hypotension on the post-ECMO $5^{\text {th }}$ day. In all patients, conventional lungprotective ventilation as mentioned before was sustained during ECMO support and maintained in the four weaned patients on the first day after ECMO cessation. The level of PEEP was gradually decreased during weaning from ECMO and afterward during weaning from mechanical ventilation. After improvement of lung functions $\left(\mathrm{FiO}_{2}\right.$ $<0.5$, PEEP $<10 \mathrm{~cm} \mathrm{H} \mathrm{H}_{2} \mathrm{O}$, peak inspiratory pressure in pressure-controlled ventilation $<25 \mathrm{~cm} \mathrm{H}_{2} \mathrm{O}$ ), ECMO flow was gradually reduced lower than $2.0 \mathrm{~L} / \mathrm{min}$.

Computed tomography scans indicated generalized ground-glass appearance and consolidations decreased.

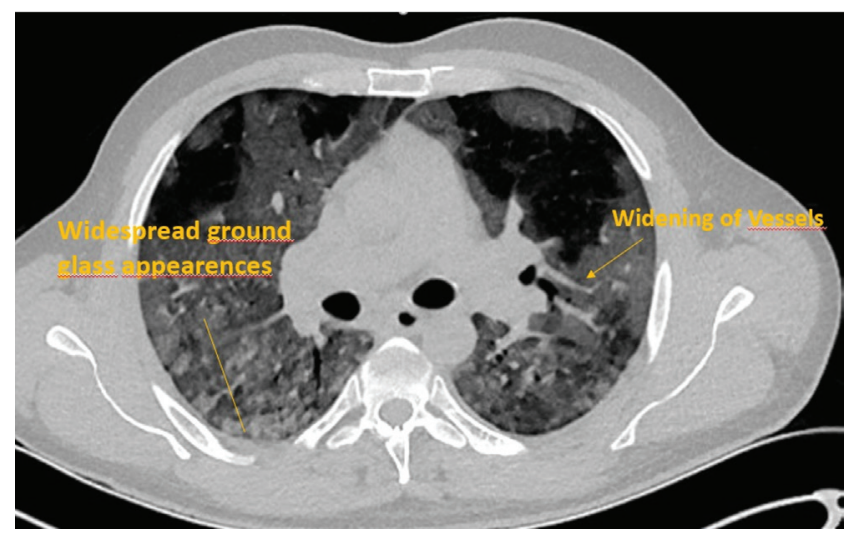

Figure 1a. Pre ECMO-Lung computerized tomography ECMO: Extracorporeal membrane oxygenation

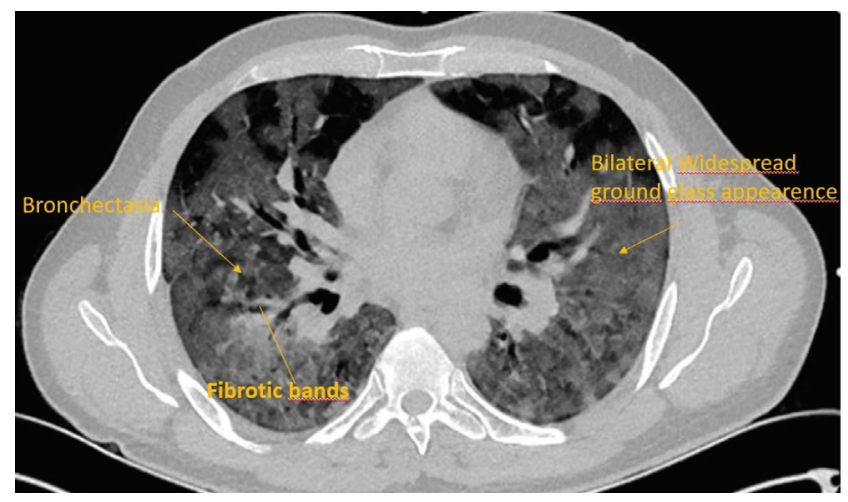

Figure $\mathbf{1 b}$. Pre ECMO-Lung computerized tomography ECMO: Extracorporeal membrane oxygenation

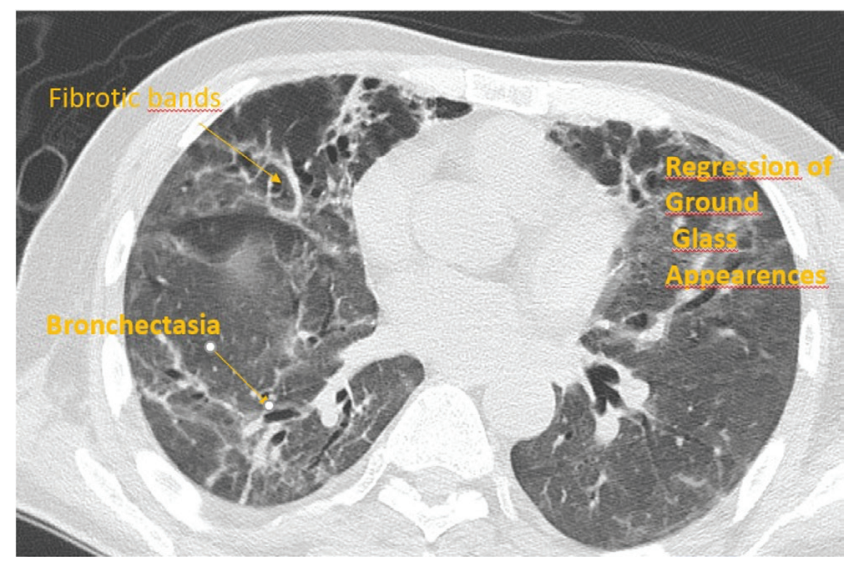

Figure 2a. After weaning off ECMO computerized tomography ECMO: Extracorporeal membrane oxygenation

Figure $1 \mathrm{a}$ and $1 \mathrm{~b}$ indicate the pre-ECMO findings and Figure $2 \mathrm{a}$ and $2 \mathrm{~b}$ are post-ECMO findings. While four of 22 patients $(18.18 \%)$ weaned off ECMO, the ratio was $22.2 \%$ (4 of 18 patients) for VV ECMO supported patients. Their blood gas values were at normal ranges but due to long intubation duration and increased secretion, three 
of them needed tracheostomy and they were discharged from the intensive care unit (ICU) to the ward with a tracheostomy cannula.

Among the 4 weaned off ECMO patients, 3 could be discharged from the hospital within the third week without any neurologic or ischemic sequela. Patients

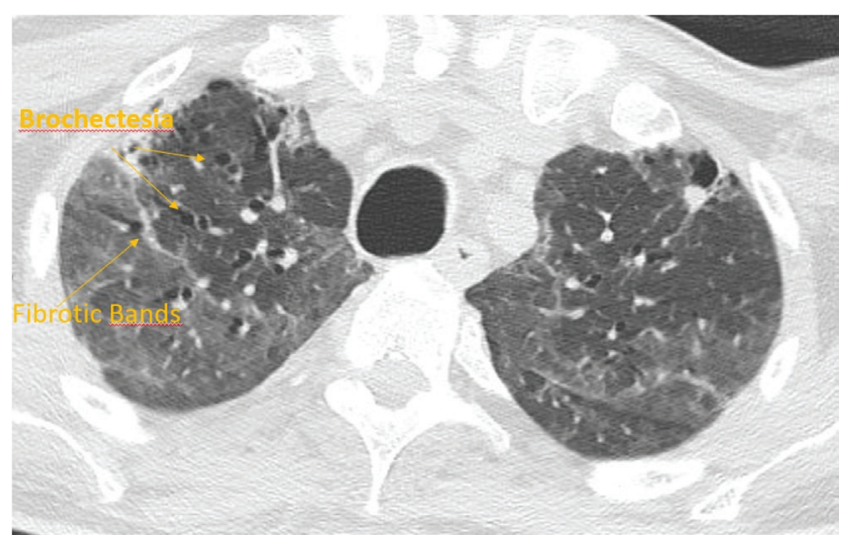

Figure $\mathbf{2 b}$. After weaning off ECMO computerized tomography ECMO: Extracorporeal membrane oxygenation scheduled for ECMO treatment research flow diaphragm is added as Figure 3.

\section{Discussion}

The pathophysiology of respiratory failure in Severe acute respiratory syndrome coronavirus-2 (SARS-CoV-2) is due to massive alveolar damage. The ARDS rate of hospitalized patients range between $15 \%$ and $30 \%(7)$. Although in patients with COVID-19 pulmonary failure is expected primarily, there may be numerous patients requiring VA ECMO support. The underlying causes may be potential primary cardiac involvement that causes arrhythmias or myocarditis and the development of consecutive circulatory failure due to increased thromboses. A combination of increased thromboses with severe systemic inflammation increases the risk of atherosclerotic plaque disruption and acute myocardial infarction $(11,12)$.

ECMO is a useful device especially in advanced cases of cardiac and respiratory failure. ECMO treatment in patients with COVID-19 is not fully established. The severity of lung damage also affects ventilation time, and immobility may restrict its benefits. All ECMO centers should have

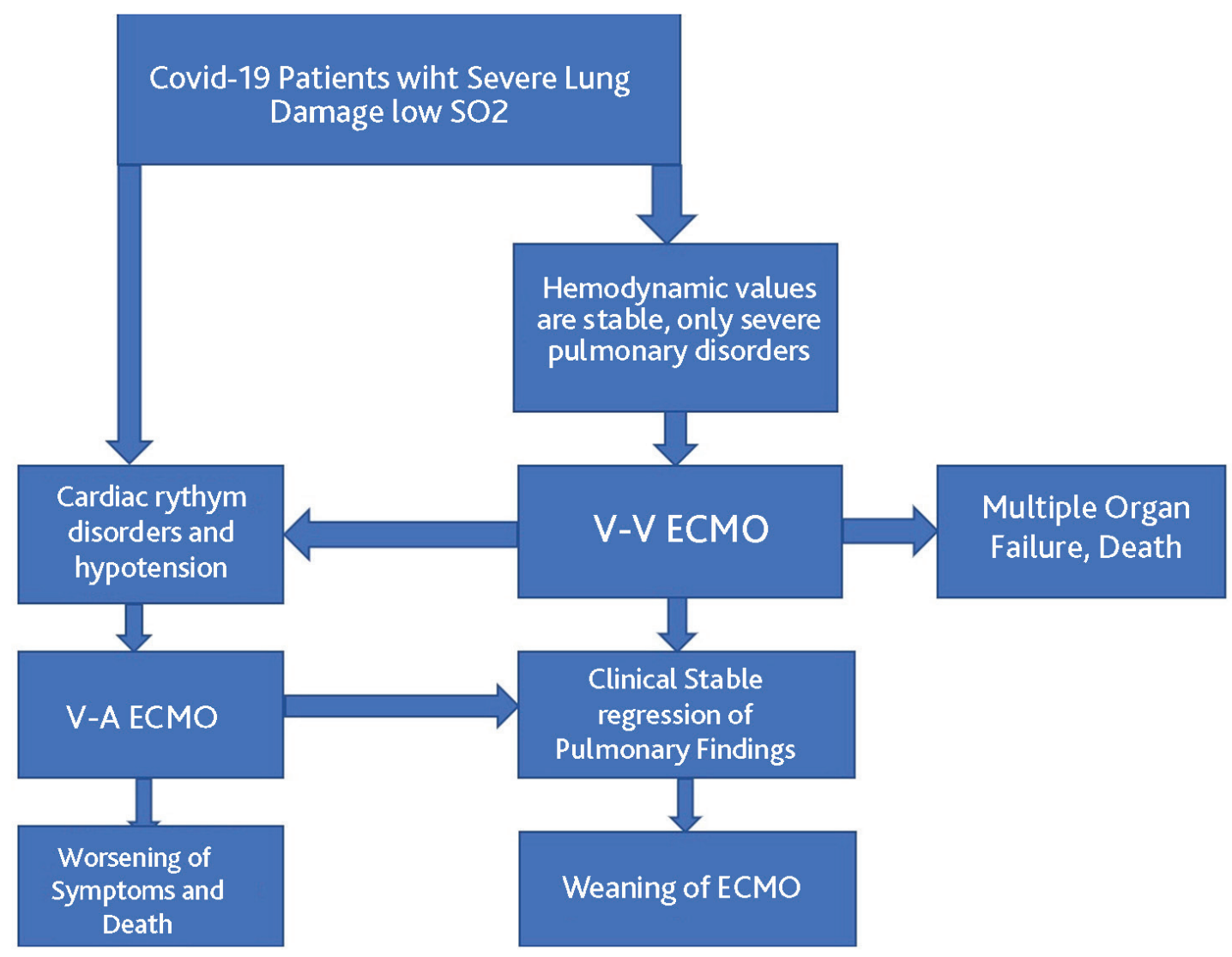

Figure 3. Research flow diaphragm

ECMO: Extracorporeal membrane oxygenation, COVID-19: Coronavirus disease-2019, VV: Veno-venous, VA: Veno-arterial 
guidelines for ECMO application and weaning off protocols, and also supplementary team members as anesthesiology, cardiovascular surgery, thoracic and respiratory experts, infectious diseases experts should be involved in the management of treatment. Following satisfying weaning off ECMO, patients should be decannulated without any major complications, and respiratory and physical rehabilitation should be implemented with the use of Personal Protective Equipment (4). Moreover, outcomes with ECMO may be affected by several variables, as the rate of recovery of the pulmonary disease, secondary infections, and the effectiveness of antiviral drugs. In addition to conventional intensive care practices and sterility and infection management protocols, careful patient selection should be among the principles of care $(3,4,13)$.

Cardiac rhythm and myocardial function evaluation have priory in the decision of VV or VA ECMO (14). In our cases, 3 of the patients had rhythm disorders and hypotension which made us decide to support these patients with VA ECMO. In one case, hypotension developed after VV ECMO support and we switched from VV to VA ECMO by cannulation of the femoral artery. Unfortunately, this patient was also lost due to cardiac rhythm disorders and septic cardiogenic shock.

Due to severe and bilateral lung damage in critically ill patients with COVID-19, patient selection for ECMO need to be careful. The factors that influence outcomes of patients like age, comorbidities, and multiple organ failure should be strictly considered especially in a situation of pandemics with limited trained personnel and sources as ECMO, ICU beds, and blood and blood products. In addition to careful patient selection, conventional intensive care management and infection control protocols should be among the primary priory of care (4). In our cases mean age of patients was $56.33 \pm 10.63$ years and the range was 30-69 years. Also, in our clinic previously known coronary artery or rhythm disorders, renal and pulmonary dysfunctions or chronic pulmonary diseases were exclusion criteria for ECMO application in this particular patient population with COVID-19 associated ARDS. All of the patients were controlled by infectious diseases and CRP and ferritin levels were checked and routine cultures as tracheal aspirate, urine, and blood were investigated if he or she had fever. Anti-biotherapy was arranged according to the culture results for secondary infections such as Staphylococcus aureus, Acinobacterium or yeast.

Chronic kidney disease is associated with progressive illness or even death in COVID-19 as a comorbidity factor. But also, ECMO and COVID-19 are independent risk factors for acute kidney failure. However, for an effective management strategy, identification of the pathophysiology underlying renal manifestations of COVID-19 is needed. Monitoring of markers of kidney functions is also helpful in the identification of patients who are at high risk for worse outcomes during ICU follow-up for COVID-19 (15). Hemodialysis and hemofiltration were performed in 19 of 22 cases in our cohort although they had no known previous renal disease and their renal markers as BUN and creatinine were at normal ranges. Due to acidosis and low blood pressure, urine output decreased and creatinine values increased, so we needed to support these patients with renal replacement therapy alternatives.

Lymphopenia is also one of the effective and reliable findings to indicate the severity and hospitalization in COVID-19 patients (1). In our cases also lymphocyte count was meaningfully lower and the mean percent of lymphocytes were 4.16 2.10 (normal range: 22-40). Together with the decrease of the symptoms, with an inverse relationship, the number of lymphocytes increased.

In our cases, we aimed to choose VV ECMO at first in contrary to VA ECMO. In VV ECMO support the patient's erythrocyte transfusion need was lower than VA ECMO. And low transfusion requirement also attenuated the transfusion-related lung injury. In another aspect, cardiac involvement also decreases the success of ECMO. In our cases, the duration of VA ECMO support was very low as patients were lost due to hypotension and serious rhythm disorders despite full ECMO support. So, we become to change our choice of patients to stable patients in the cardiac aspect.

Some of the limitations of outcomes in ECMO applied COVID-19 patients are increased immobility and catheter infections and for this reason the use of the right internal jugular vein via dual-stage cannula as a single-access. The advantages of this cannula are the direct arterial flow to right ventricle and then to the pulmonary artery, thus we may achieve better oxygenation and ventilation; more easily giving position in bed and early mobilization. Additionally, a single cannula also decreases the risks of complications or revisions (16). In this research, single-access, dual-stage cannula was chosen in two cases but, in these patients required adequate support for blood oxygenation could not be reached and the patient's oxygen saturation could not be increased above $90 \%$. So, one of the patients was lost due to multi-organ failure and in another patient, we decided to switch cannulas to femoral and internal jugular veins and his blood gas measurements became to improve and he was weaned off ECMO. While four of 22 patients (18.18\%) weaned off ECMO, this ratio was $22.2 \%$ (4 of 18 patients) for VV ECMO-supported patients and 3 of them 
could be discharged from the hospital. Other patients were cannulated from the right internal jugular vein and the right femoral vein with ultrasonography guidance and we did not face with any complications with this catheterization strategy.

\section{Study Limitations}

The study has certain limitations. The retrospective nature of the research is one of the limitations. Another limitation is the relatively small cohort size. The COVID-19 affects multiple organs and the effects of ECMO cannot be predicted exactly as being retrospective with low patient numbers as in our small cohort sample. However, our aim was to present our single-center clinical experiences in this unique group of COVID-19 patients requiring ECMO.

\section{Conclusion}

Although, ECMO support for severe respiratory failure patients with COVID-19 is more challenging than regular ECMO applications, especially VV ECMO utilization yielded better outcomes and should be kept in mind as a remedy. The decision and application should be based on a teamwork approach to carefully choose the most suitable patients who will benefit the most from this invasive treatment option for better outcomes and increased success rates as well as in order not to harm otherwise recovering patients with conventional care measures.

\section{Authorship Contributions}

Concept: M.O.U., Design: A.K., Data Collection or Processing: N.K., Y.Y., Analysis or Interpretation: M.U., S.O., Literature Search: K.E., Writing: M.O.U., D.M.O.

Conflict of Interest: No conflict of interest was declared by the authors.

Financial Disclosure: The authors declared that this study received no financial support.

\section{References}

1. Yavuz BG, Colak S, Güven R, Urun A, Satilmis D, Altundag I. A Survey Analyzing Reflections of COVID-19 on Patients and Their Relatives. Med Bull Haseki 2021;59:1-8.

2. Zhou F, Yu T, Du R, et al. Clinical course and risk factors for mortality of adult inpatients with COVID-19 in Wuhan, China: a retrospective cohort study. Lancet 2020;395:1054-62.

3. Hartman ME, Hernandez RA, Patel K, et al. COVID-19 Respiratory Failure: Targeting Inflammation on VV-ECMO Support. ASAIO J 2020;66:603-6.
4. Ramanathan K, Antognini D, Combes A, et al. Planning and provision of ECMO services for severe ARDS during the COVID-19 pandemic and other outbreaks of emerging infectious diseases. Lancet Respir Med 2020;8:518-26.

5. Tan L, Wang Q, Zhang D, et al. Lymphopenia predicts disease severity of COVID-19: a descriptive and predictive study. Signal Transduct Target Ther 2020;5:33.

6. Ugurlucan M, Yildiz Y, Oztas DM, et al. Congenital cardiac interventions during the peak phase of COVID-19 pandemics in the country in a pandemics hospital in Istanbul. Cardiol Young 2020;30:1288-96.

7. Wang D, Hu B, Hu C, et al. Clinical Characteristics of 138 Hospitalized Patients With 2019 Novel Coronavirus-Infected Pneumonia in Wuhan, China. JAMA 2020;323:1061-9.

8. Yildiz Y, Oztas DM, Ulukan MO, et al. Application of Hybrid Extracorporeal Membrane Oxygenation for the Treatment of Subsequent Shock following Acute Respiratory Distress Syndrome Developing after Firearm Injury. Case Rep Med 2019;2019:3120912.

9. Loforte A, Dal Checco E, Gliozzi G, et al. Veno-venous Extracorporeal Membrane Oxygenation Support in COVID-19 Respiratory Distress Syndrome: Initial Experience. ASAIO J 2020;66:734-8.

10. Yildiz A, Yigit A, Benli AR. The prognostic role of Charlson comorbidity index for critically ill elderly patients. The European Research Journal 2020;6:67-72.

11. Long B, Brady WJ, Koyfman A, Gottlieb M. Cardiovascular complications in COVID-19. Am J Emerg Med 2020;38:15047.

12. Olmez $H$, Tosun $M$, Ünver $E$, Dogan $M$, İsik NM. COVID-19 and hypercoagulability. Eur Res J 2021;7:209-17.

13. Uzman S, Ayazoglu TA. SARS-CoV-2 Infection in Intensive Care Unit Healthcare Workers in Turkey: A Tertiary Center Cohort Study. Med Bull Haseki 2021;59:103-7.

14. Wiedemann D, Bernardi MH, Distelmaier $\mathrm{K}$, ET AL. Recommendations for extracorporeal membrane oxygenation (ECMO) in COVID-19 patients : Consensus paper of the Medical University of Vienna. Wien Klin Wochenschr 2020;132:671-6.

15. Kunutsor SK, Laukkanen JA. Renal complications in COVID-19: a systematic review and meta-analysis. Ann Med 2020;52:345-53.

16. Liu JY, Merkow RP, Cohen ME, et al. Association of Weekend Effect With Recovery After Surgery. JAMA Surg 2020;155:98890. 